\title{
Combining Multi-Variate Statistics and Dempster-Shafer Theory for Edge Detection in Multi-Channel SAR Images
}

\author{
D. Borghys and C. Perneel \\ Royal Military Academy, Signal \& Image Centre \\ Av. de la Renaissance 30, B-1000 Brussels \\ Dirk.Borghys@elec.rma.ac.be
}

\begin{abstract}
A new scheme for detecting edges in multi-channel SAR images is proposed ${ }^{1}$. The method is applied to a set of two full-polarimetric SAR images, i.e. a P-band and an L-band image. The first step is a lowlevel edge detector based on multi-variate statistical hypothesis tests. As the spatial resolution of the two SAR bands is not the same, the test is applied to the polarimetric information for each band separately. The multi-variate statistical hypothesis test is used to decide whether an edge of a given orientation passes through the current point. The test is repeated for a discrete number of orientations. Eight orientations are used. The response for the different orientations of the scanning rectangles as well as for different bands is combined using a method based on Dempster-Shafer Theory. The proposed scheme was applied to a multichannel E-SAR image ${ }^{2}$ and results are shown and evaluated.
\end{abstract}

\section{Introduction}

Synthetic Aperture Radar (SAR) image products are very important and useful for remote sensing applications because they can be acquired independent of time of day or weather conditions and because their characteristics (wavelength, polarisation, observation angle) can be chosen in function of the phenomenon under investigation. The first satellite-based SAR systems used for remote sensing were single-band mono-polarisation systems with a spatial resolution of a few tens of meters (e.g. 25m for ERS1, 30m for Radarsat). However, scene interpretation results can be greatly enhanced by combining different SAR images [1] e.g. multi-polarisation, multi-frequency, different aspect angles, multi-temporal, etc. In future satellite systems, the spatial resolution will be improved to a few meters and the systems will be capable to acquire high-resolution polarimetric and/or multi-frequency, i.e. multi-channel, data. Current airborne SAR systems are already capable to acquire multi-channel SAR images with a metric resolution. For the automatic interpretation of such images, adequate low-level image

\footnotetext{
1 The presented research is done in the frame of a European project IST-2000-25044: SMART (Space and Airborne Mined Area Reduction Tools)

${ }^{2}$ The test image was provided to us by the German Aerospace Center (DLR).
} 
processing tools are needed. In this paper we propose an edge detection scheme for multi-channel SAR images. Current edge detectors were designed to work on low-resolution, single-band, multi-look SAR images. The most widely used edge detector for such SAR images is the ratio-detector [2]. It is based on the speckle distribution in uniform regions in single-band multi-look intensity images. In $[3, ?]$ we proposed new edge detectors for polarimetric SAR images and based on multi-variate statistical hypothesis tests. The hypothesis test is applied for different orientations of a set of two scanning rectangles. In order to determine whether a vertical edge passes through a point $P$ two vertical rectangles are constructed around the point $\mathrm{P}$ and the statistics of the pixels in both rectangles are compared using the hypothesis test. The test is repeated for a given number of different orientations of the scanning rectangles. Normally the maximum of the response over all orientations is considered as the global edge response. In this article we investigate a new and improved way to combine (fuse) the responses of statistical edge detectors. The method is based on Dempster-Shafer evidence theory $[5, ?]$ which is briefly described in section 3 . In a first step the fusion method is applied to combine the response of the statistical test over the different orientations of the scanning rectangles. In a second step the same fusion method is used to combine edge detection results obtained from the two frequency-bands. In section 2 the edge detector based on multi-variate statistical hypothesis is introduced, section 3 gives a brief summary of Dempster-Shafer evidence theory which is applied to the fusion of edge detection results in section 4. In section 5 results of applying the method on a set of two polarimetric SAR images, are shown and discussed. The last section presents the conclusions and the perspectives for further research.

\section{An Edge Detector based on Multi-Variate Statistics}

An obvious way to detect edges in multi-channel images is to fuse the results of existing detectors applied on each individual channel. An alternative is to use multi-variate statistical methods which treat the combined information from the different channels as a single input-vector. We have already successfully introduced such methods for detecting edges in polarimetric SAR images $[3, ?]$. Fig. 1 illustrates the two approaches that can be used for detecting edges in multichannel SAR images. A comparative evaluation [7] has shown that the multivariate methods outperform the fusion of uni-variate methods. The multi-variate hypothesis test for equality of variances that was used is the Levene test [8]. It is applied to the single-look complex data where differences in radar backscattering appear as differences in variance of a zero-mean normal distribution. The nullhypothesis $H_{o}$ is that the samples from the two scanning rectangles are from populations with the same variance, the alternative hypothesis $H_{1}$ is that the population variances are different. In the Levene test the samples from the two scanning windows are first transformed in absolute deviations of sample means. In the case of a single-look complex polarimetric image with complex data of 


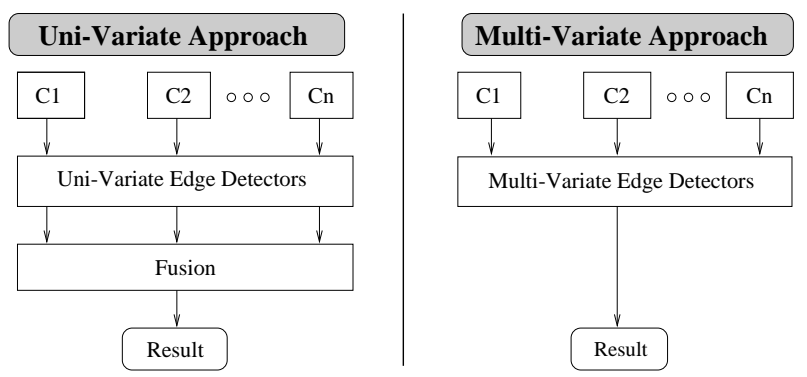

Fig. 1. Edge Detection in Polarimetric SAR

the type $x^{H H}, x^{H V}, x^{V V}$ this results in:

$$
\mathbf{L}_{i k}=\left[\left|\Re\left(x_{i k}^{H H}-\bar{x}_{k}^{H H}\right)\right|,\left|\Im\left(x_{i k}^{H H}-\bar{x}_{k}^{H H}\right)\right|, \ldots,\left|\Im\left(x_{i k}^{V V}-\bar{x}_{k}^{V V}\right)\right|\right]^{t},
$$

in which $\mathrm{i}$ is the index of the observations and $\mathrm{k}$ the index of the scanning window ( $k=1$ or 2 ). The question whether two samples display significantly different amounts of variance is then transformed into a question of whether the transformed values show a significantly different mean. This can then be tested using a Hotellings $T^{2}$-test $[9,8]$. The Hotellings $T^{2}$-statistic is defined as:

$$
T^{2}=\frac{n_{1} n_{2}\left(\overline{\mathbf{L}_{\mathbf{1}}}-\overline{\mathbf{L}_{\mathbf{2}}}\right)^{t} \mathbf{C}^{-1}\left(\overline{\mathbf{L}_{\mathbf{1}}}-\overline{\mathbf{L}_{\mathbf{2}}}\right)}{n 1+n 2},
$$

with $\overline{\mathbf{L}_{\mathbf{k}}}$ the average of the $\mathbf{L}_{i k}$ values in the $k^{t h}$ window and $[C]$ the pooled covariance matrix estimated by:

$$
\mathbf{C}=\frac{\left(n_{1}-1\right) \mathbf{C}_{\mathbf{1}}+\left(n_{2}-1\right) \mathbf{C}_{\mathbf{2}}}{n_{1}+n_{2}-2},
$$

where $C_{1}$ and $C_{2}$ represent the covariance matrices estimated from the two scanning rectangles. The significance of $T^{2}$ is determined by using the fact that in the null-hypothesis of equal population means the transformed statistic

$$
T_{F}=\frac{\left(n_{1}+n_{2}-p-1\right) T^{2}}{\left(n_{1}+n_{2}-2\right) p}
$$

follows a Fisher-Snedecor distribution, $F_{\nu_{1}, \nu_{2}}$ with degrees of freedom $\nu_{1}=p$ and $\nu_{2}=n_{1}+n_{2}-p-1 . \mathrm{p}$ is the number of variants, i.e. 6 in our case if the real and imaginary components for each polarisation are counted separately. From the theoretical distribution of the test statistic the theoretical $\alpha \%$ false alarm threshold $\theta_{\alpha}$ for the detector can be determined. It is given by

$$
P\left\{T_{F} \geq \theta_{\alpha} \mid H_{o}\right\}=\alpha \text {. }
$$

The theoretical distribution of the test-statistic when the null-hypothesis is verified is used to transform the test-statistic in each point in the image into the 
corresponding $\mathrm{p}$-value. For a value pf the test-statistic $T_{F}(x, y)$, found in a given pixel, the p-value is the probability that an even more extreme value can be found when the null-hypothesis is verified. A low p-value means the test-statistic is very extreme and indicates that the null-hypothesis is probably not verified, i.e. the region corresponding to the two scanning rectangles is not uniform, and there might be an edge passing between the two rectangles. Using p-values allows to compare and combine results of different edge operators.

\section{Overview of the Dempster-Shafer Theory Framework}

The aim of the fusion described in the current article is to combine the response of the edge detector for different orientations of the scanning rectangles as well as to combine the results obtained in different SAR images of the same scene. The proposed fusion method is based on Dempster-Shafer (DS) theory $[5, ?]$. Dempster-Shafer or evidence theory is a mathematical tool that allows to work with uncertain, imprecise and incomplete information. The uncertainty is taken into account by assigning masses to sets of different hypotheses. Several experts distribute their knowledge over these different hypotheses and a final decision is obtained after combining the masses assigned by each expert. In DS-theory a set of hypotheses is defined: $\Theta=\left\{H_{1}, H_{2}, \ldots H_{n}\right\}$. The different experts or sources of information distribute masses to sub-sets $A_{i}$ of $\Theta$. For each source of information a mass function is defined as:

$$
\begin{aligned}
m: & 2^{\theta} \rightarrow[0,1] \\
& A_{i} \rightarrow m\left(A_{i}\right),
\end{aligned}
$$

in which $2^{\theta}$ is the set of all sub-sets of $\Theta$ and $m\left(A_{i}\right)$ represents the confidence that the information source has that the solution lies in the sub-set $A_{i}$. The attribution of masses for each information source is constrained by the following rules:

$$
\begin{aligned}
& 0 \leq m\left(A_{i}\right) \leq 1, \\
& m(\Phi)=0, \\
& \sum_{A_{i} \in 2^{\Theta}} m\left(A_{i}\right)=1,
\end{aligned}
$$

where $\Phi$ denotes the empty set. The solution is found by combining the masses attributed to the different sub-sets by the different experts. The combination of masses from different experts is done by Dempster's combination rule. Let $m_{1}$ and $m_{2}$ be the masses that were respectively attributed by expert 1 and expert 2 , then the combination of the masses from these two is defined as:

$$
m_{12}\left(A_{i}\right)=\sum_{A_{p} \cap A_{q}=A_{i}} m_{1}\left(A_{p}\right) m_{2}\left(A_{q}\right) .
$$

Masses are thus attributed to the sub-set formed by the intersection of the different sub-sets. Depending on what happens when the intersection is empty, one distinguishes the closed world or the open world model. In the closed world one assumes that the solution corresponds necessarily to one of the defined sub-sets. 
Any mass that would be combined into the empty set is therefore redistributed over all other sets and the mass of the empty set remains zero. In the open world model one allows the possibility that the solution is not part of the defined subsets. A mass that goes into the empty set can then be interpreted as a symptom of the fact that the solution is not within the sub-sets or that different experts have incompatible opinions. From the combined masses two functions can be derived that characterise the support to the final decision. The first is called the belief $(\mathrm{Bel})$ and represents the degree of minimal support on sub-set $A_{i}$. The second is called plausibility $(P l s)$ and corresponds to the maximal or potential support to a given sub-set in the final mass assignment [6]. They are defined as:

$$
\operatorname{Bel}\left(A_{i}\right)=\sum_{A_{j} \subseteq A_{i}} m\left(A_{j}\right) \quad, \quad P l s\left(A_{i}\right)=\sum_{A_{j} \cap A_{i}} m\left(A_{j}\right) .
$$

In the design of a system for fusion of information based on DS-theory one distinguishes the following steps:

- Define the sub-sets relative to the problem

- Choose the model (closed or open)

- Define the mass functions used by each expert to distribute its confidence to the different sets

\section{Application of DS-Theory to the Fusion of Edge Detection Results}

\subsection{Definition of the Sub-Sets and the Strategy}

The aim is to combine the response of the edge detector for different orientations of the scanning rectangles. The edge detector for each orientation of the windows is considered as an expert giving its opinion about the presence of an edge along that direction. A small p-value means the expert has a strong opinion about the presence of the edge and consequently a high confidence should be given to that direction. The larger the p-value, the less strong the opinion is and the less confidence should be given to that particular direction. We use 8 orientations $D_{0} . . D_{7}$ (ranging from $D_{0}=0$ to $D_{7}=157.5^{\circ}$ in steps of $22.5^{\circ}$ ) of the scanning rectangles and we say that if a low p-value is found for a given direction, it does not necessarily mean an edge is located along that direction; it could be oriented along neighbouring directions. Even when we find an edge in a given orientation, we do not know whether there is, in the same point not also an edge along another orientation (a corner). Therefore we need to attribute also some mass to the other directions. We have defined the following sub-sets of directions:

- the singleton: $\left\{D_{i}\right\}$,

- the triplet: $\left\{D_{i-1}, D_{i}, D_{i+1}\right\}$,

- the complement of the direction $\overline{\left\{D_{i}\right\}}$

- the complete set of directions $\left\{D_{0} . . D_{7}\right\}$ 
The open world model is the most convenient for our problem [13]. In general the mass of the empty set $m(\Phi)$ after combining the masses is an indication for a disagreement between experts or for the fact that the solution is not among the defined sub-sets. This mass should therefore be high at corners The mass of the complete set $m_{C}$ indicates an indecisiveness of the experts. It will be low in edges or corners and high in the background. Three cases are distinguished:

- Background: If all p-values are "high", probably no edge is present, and we attribute most of the mass to the complete set of orientations, i.e. we know nothing to decide the orientation of an edge. The complete set will, when the masses are combined over different experts, not contribute to the mass of the empty set. Therefore the mass of the empty set will be very low.

- Corners: Here several experts may detect an intermediate p-value and we should find a high conflict between the experts and the mass of the empty set should be high.

- Edges: The p-value for the correct edge direction is very low while neighbouring directions will also have a low p-value. The mass of the complete set should be low because some experts are very sure, while there is some conflict due competing neighbouring directions.

\subsection{Learning the System's Parameters}

For determining the system's parameters a learning set with examples of edge (EP), corner (CP) and background points (BP) was selected.

Thresholds for the p-Values. In order to introduce a dependence of the mass assignment on the p-values that are obtained for the different orientations, the range of possible p-values was sub-divided into 5 sub-ranges corresponding to increasing $\mathrm{p}$-value. The actual borders are fixed by studying the p-values of the set of learning points for a given edge direction. The thresholds are selected such that for the correct edge direction the p-values are very low or low; for corners they are intermediate or high and in the background the p-values are high or very high. The p-value thresholds that gave the best results for the Levene test are: $T_{1}=10^{-8}, T_{2}=10^{-7}, T_{3}=10^{-4}, T_{4}=10^{-2}$.

Optimisation of the Mass Functions. Even when the sub-sets are chosen and when we know what should be the result of the combination of masses from different experts, it is still difficult to design the mass functions consequently. We therefore determined the mass functions automatically on the basis of a small learning set. In order to find the optimal mass functions a cost function $C_{t o t}=C_{\Phi}+C_{C}$ is defined as the sum of a cost function defined on the empty set and the complete set as:

$C_{\Phi}=\frac{1}{N_{B P}} \sum_{p \in B P}\left[m_{\Phi, p}-0.1\right]^{2}+\frac{1}{N_{E P}} \sum_{p \in E P}\left[m_{\Phi, p}-0.5\right]^{2}+\frac{1}{N_{C P}} \sum_{p \in C P}\left[m_{\Phi, p}-0.9\right]^{2}$, 
$C_{C}=\frac{1}{N_{B P}} \sum_{p \in B P}\left[m_{C, p}-0.5\right]^{2}+\frac{1}{N_{E P}} \sum_{p \in E P}\left[m_{C, p}-0.1\right]^{2}+\frac{1}{N_{C P}} \sum_{p \in C P}\left[m_{C, p}-0.1\right]^{2}$.

The masses of the different sub-sets are adapted iteratively in order to minimise the cost function $C_{t o t}$ on the learning points. The optimisation is performed using the downhill simplex method of Nelder and Mead [10,11]. Convergence is reached after 20 to 25 iterations. The resulting mass functions are shown in table 4.2. The general tendency for the resulting mass functions after optimisation is that for very low p-values most of the mass goes to the singleton. As $\mathrm{p}$-value increases the mass of the triplet (neighbouring edge orientations) and the complement (a possible indication of corners) increases and finally, for very high p-values, most of the mass is concentrated in the complete set which corresponds to undeciveness (background). Masses in the table correspond to the value assigned when the p-value corresponds to the given thresholds; for p-values in between the thresholds, masses are linearly interpolated.

Table 1. Mass functions after optimisation of cost function

\begin{tabular}{c|c|c|c|c|c|c}
\hline \multirow{2}{*}{ Sub-Set } & \multicolumn{5}{|c}{ Threshold } \\
\cline { 2 - 7 } & 0.0 & $T_{1}$ & $T_{2}$ & $T_{3}$ & $T_{4}$ & 1.0 \\
\hline$\left\{D_{i}\right\}$ & 0.497 & 0.473 & 0.014 & 0.101 & 0.018 & 0.021 \\
$\left\{D_{i-1}, D_{i}, D_{i+1}\right\}$ & 0.174 & 0.062 & 0.906 & 0.131 & 0.010 & 0.089 \\
$\left\{D_{i}\right\}$ & 0.066 & 0.329 & 0.005 & 0.299 & 0.000 & 0.009 \\
$\left\{D_{0} . D_{7}\right\}$ & 0.262 & 0.136 & 0.075 & 0.470 & 0.972 & 0.882 \\
\hline
\end{tabular}

\subsection{Fusion of Results from Different Frequency Bands}

The test set used in this paper consists of two polarimetric images, respectively in P-band and L-band. The two images were acquired from two parallel flight paths and cover approximately the same region. However the spatial resolution of both images is not the same. Together with the SLC images we received transformation matrices that enable one to find the ground coordinates of each point in the SLC images. These were obtained by the German Space Agency DLR by geocoding the SAR image using a DEM of the region. By combining the transformation matrices from the two bands it is thus possible to find the relationship between the positions in the two images. However, because of the difference in spatial resolution, this is not a one-to-one relationship. This is why we decided to apply the raw edge detector to each band separately and fuse only the results of the edge detection. In this paper we used the two images as two sets of experts voting for a given edge orientation in each pixel of the P-band image. The DS-based fusion is used to combine the different experts for different edge orientations as well as for the two bands. As a reference, the image with lowest resolution is used, i.e. the $\mathrm{P}$-band image. The $\mathrm{P}$-band image is scanned 
and in each pixel first the edge information from the different orientation experts is gathered and then the corresponding point in the L-band image is determined and the edge information from the L-band image at that point is also gathered. The DS-fusion is used to combine this joint information.

\section{$5 \quad$ Results and Discussion}

\subsection{Fusion of Edge Orientations}

In fig. 2 a part of the original P-band E-SAR image is shown on the left. The edge detector is applied to the three polarisations simultaneously. The dimension of the scanning rectangles is $10 \times 50$. The 2 nd and 3 rd image respectively show the mass of the empty set $m_{\Phi}$ and of the complete set $m_{C}$ after combination of masses. High values for $m_{\Phi}$ correspond to corners and to other locations in the
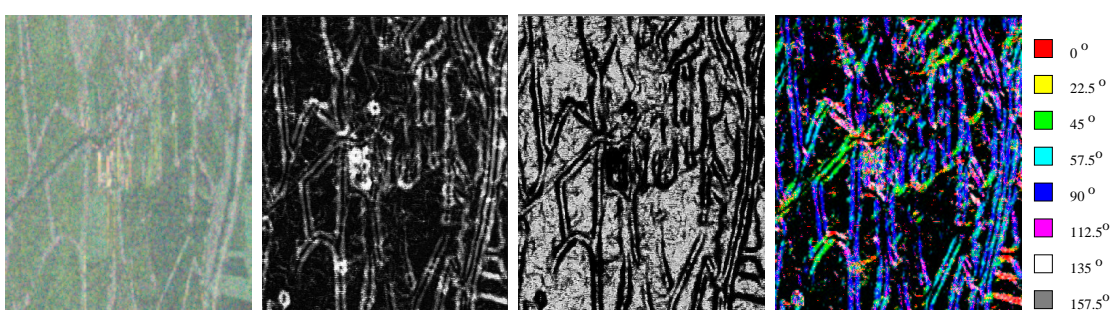

Fig. 2. Pband image results (from left to right: original image, $m_{\Phi}, m_{C}$ and image of edge orientations)

image with high uncertainty with respect to the orientation of edges, e.g. highly textured regions (built-up areas, forests or lines of trees). On the other hand $m_{C}$ is low at the position of edges and corners. The decision whether a given point of the image corresponds to an edge (or corner) can thus be based on the combined information in $m_{\Phi}$ and $m_{C}$. If the point belongs to an edge, the orientation of the edge can be derived from the plausibility and the belief. The orientation corresponds to the singleton of directions for which the highest plausibility is found. The right image of fig. 2 represents the image of edge orientations.

\subsection{Fusion of Edge Detection Results from the Two Bands}

In fig. 3 the results of fusing the edge information of the P-and L-band image are shown. Note that the L-band image in the figure was geometrically rescaled to the same size as the P-band image for display purposes. Results are also shown in the coordinates of the P-band image. Note that the image of the complete set shows more edge detail after the fusion. 

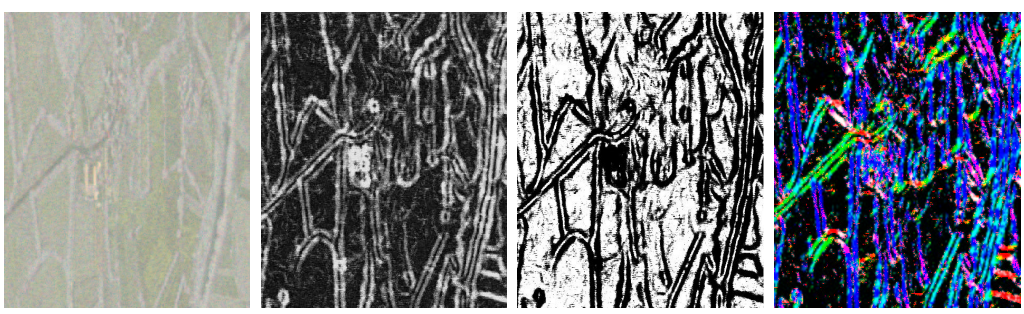

Fig. 3. Results of fusing the edge information of the two frequency bands (from left to right: original L-band image, results after fusion for $m_{\Phi}, m_{C}$ and edge orientation)

\subsection{Comparative Evaluation of the Results}

The images above already show that the results after fusion of the two bands are better than without the fusion. In order to obtain a quantitative idea of the detector's performance we determined the Receiver-Operator Characteristic (ROC) curves for edge detection based on the P-band alone and after fusion of P-band and L-band. ROC curves show the probability of detection $P_{d}$ of a detector versus its probability of false alarms $P_{f} . P_{d}$ and $P_{f}$ are determined on a test image in which the true edges are known. These "true" edges were indicated manually on the image. The ROC curve is generated by varying the detector's threshold. Fig. 4 shows the ROC curves obtained with and without fusion of the two bands. The curve found for the fusion is above the curve found for the $\mathrm{P}$-band, indicating that the combination of the two bands indeed improves edge detection results.

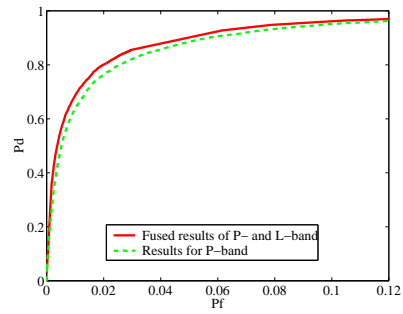

Fig. 4. ROC curve for edge detection results

\section{Conclusions and Perspectives}

In this article a new scheme for detecting edges in multi-frequency polarimetric SAR images is presented. It consists of two steps. The first step uses a multivariate statistical hypothesis test to decide whether an edge of a given orientation passes through the current point. The test is repeated for a discrete number (8) 
of orientations. The multi-variate test is applied to the full-polarimetric image, but each frequency band is treated separately because their spatial resolution is different. The second step combines the edge detector response over the different orientations. For this combination an approach based on Dempster-Shafer theory was developed. The edge detector for each orientation behaves as an expert that gives an opinion about the presence of an edge in a set of possible directions. The confidence each expert assigns to each sub-set of orientations is determined using mass function. A cost function is defined to find masses in order to increase the distinction between edges, corners and background. The masses are automatically optimised using this cost function. The method is applied to a set of two full-polarimetric E-SAR images in resp. P- and L-band. In a next step we will investigate further how to incorporate local spatial information, i.e. taking into account neighbours of each pixel, to improve edge detection. In particular we will explore a method to increase further the confidence in a given edge pixel when neighbouring edge pixels in the higher-resolution image are found along the same edge direction. We will also investigate the use of active contours to improve detected edge structure and investigate synergy between our edge detector and speckle reduction methods.

\section{References}

1. Ulaby, F.: Sar biophysical retrievals: Lessons learned and challenges to overcome. In: Retrieval of Bio- and Geophysical Parameters from SAR Data for Land Applications, ESTEC, NL (1998)

2. Touzi, R., Lopes, A., Bousquet, P.: A statistical and geometrical edge detector for sar images. IEEE-GRS 26 (1988) 764-773

3. Borghys, D., Perneel, C., Acheroy, M.: Contour detection in high-resolution polarimetric sar images. In: SPIE Conf. on SAR Image Anal. ,Modelling and Tech. III; Barcelona. (2000)

4. Borghys, D., Perneel, C., Acheroy, M.: A multi-variate contour detector for highresolution polarimetric sar images. In: Proc. ICPR 2000, Barcelona. Volume 3. (2000) 650-655

5. Dempster, A.: A generalisation of bayesian inference. Journal of the Royal Statistical Society 30 (1968) 205-247

6. Smets, P.: Belief functions. Technical Report TR/IRIDIA/89-4, IRIDIA, Universite Libre de Bruxelles, Brussels (1989)

7. Borghys, D.: Interpretation and Registration of High-Resolution Polarimetric SAR Images. PhD thesis, ENST E 031, Paris (2001)

8. Manly, B., ed.: Multivariate Statistical Methods. Chapman and Hall (1995)

9. Anderson, T.: Introduction to Multivariate Statistical Analysis. J. Wiley \& Sons (1958)

10. Press, W.: Numerical Recipes in C. Cambridge University Press (1992)

11. Coleman, T.: Optimization Toolbox for use with MATLAB. Matworks Inc. (1999)

12. Fjörtoft, R.: Segmentation d'images radar par detection de contour. PhD thesis, Institut National Polytechnique de Toulouse, Toulouse (1999)

13. Neveu, S., Boivin, V.: Contribution à la détection de contours sur des images sar: fusion de données par la théorie de dempster-shafer. Projet de fin détudes, Ecole Navale et Groupe des Ecoles Poulmic, Brest (2002) 